\title{
Manejo perioperatorio de los anticoagulantes orales de acción directa en procedimientos invasivos-quirúrgicos programados. Historia de un claroscuro
}

\author{
Perioperatory management of direct action oral anticoagulants \\ in programmed invasive-surgical procedures
}

\author{
Manuel Jesús Núñez-Fernánez ${ }^{1}$, José Antonio Díaz-Peromingo² \\ ${ }^{1}$ Servicio de Medicina Interna, Complejo Hospitalario Universitario de Pontevedra. ${ }^{2}$ Servicio de Medicina Interna, ComplejoHospitalario Universitario de Santiago de \\ Compostela
}

\section{RESUMEN}

En la actualidad los pacientes tratados con anticoagulantes orales de acción directa (AOAD) son un grupo poblacional cada vez más frecuente en nuestros centros sanitarios. Las recomendaciones de manejo de estos pacientes, que van a ser sometidos a pruebas invasivas-diagnósticas 0 quirúrgicas con carácter programado, está recogidas en guías de práctica clínica y revisiones. Sin embargo hasta la fecha, todas las guías tienen el grado de evidencia de recomendación de expertos. Recientemente con la publicación del estudio PAUSE las recomendaciones existentes probablemente cambien. En esta revisión se hace mención a diferentes aspectos en relación con el manejo perioperatorio de los pacientes tratados con $A O A D$ y a las controversias existentes.

Palabras clave: Anticoagulantes orales de acción directa; Procedimientos perioperatorios; Dabigatran; Rivaroxaban; Estudio Pause

\section{INTRODUCCIÓN}

Los datos obtenidos de aseguradoras y registros sobre anticoagulantes orales de acción directa (AOAD) confirman la utilización de estos fármacos (dabigatran, rivaroxaban, apixaban, edoxaban) de una forma que aumenta año tras año, hasta tal punto de que en la mayoría de los países los pacientes tratados con warfarina y acenocumarol quedarán como testimoniales ${ }^{1-4}$. Después de los resultados de los diferentes ensayos clínicos, las guías recomiendan con un grado de evidencia alto, el tratamiento anticoagulante con AOAD en fibrilación auricular no valvular (FANV) y enfermedad tromboembólica venosa $(E T V)^{5,6}$.

Existen gran número de estudios epidemiológicos realizados en diferentes países, donde se confirma que tanto la FANV, como la ETV presentan una incidencia y una prevalencia que aumentan al mismo tiempo que lo hace la edad de la población estudiada ${ }^{7-9}$. Las previsiones para los próximos 25 años sobre crecimiento poblacional, tanto en Europa como en el resto del mundo, son que se modifique de forma clara la morfología de la pirámide de edades, aumentando significativamente el grupo de edad que engloba a los ancianos ${ }^{10}$. Se deduce de lo referido que atender a pacientes tratados con AOAD será cada vez más habitual por las patologías asociadas a la vejez (FANV y ETV); pacientes que en su atención médico-quirúrgica van a ser sometidos a procedimientos diagnósticos y terapéuticos invasivos, así como cirugía urgente y programada. Existen estimaciones donde se calcula

\begin{abstract}
Currently, patients treated with direct-acting oral anticoagulants (DAOAs) are an increasingly frequent population group in our health centres. The management recommendations of these patients, which are going to be subjected to invasive-diagnostic or surgical tests on a scheduled basis, are included in clinical practice guidelines and reviews. However, to date, all guides have the degree of evidence of expert recommendation. Recently with the publication of the PAUSE study the existing recommendations are likely to change. This review refers to different aspects in relation to the perioperative management of patients treated with DAOAs and the existing controversies.
\end{abstract}

Keywords: Direct Oral Anticoagulants; Perioperative procedures; Dabigatran; Rivaroxaban; Pause study

que 1 de cada 6 pacientes con fibrilación auricular precisará de manejo perioperatorio ${ }^{11}$. No existen estimaciones similares en pacientes diagnosticados de ETV y a tratamiento anticoagulante. La presente revisión pretende situar la evidencia actual relativa al manejo de los AOAD en pacientes que van a requerir de la realización de procedimientos diagnósticos invasivos 0 cirugías programadas.

\section{PROCEDIMIENTOS INVASIVOS-QUIRÚRGICOS PROGRAMADOS}

En el manejo perioperatorio de pacientes tratados con AOAD y sometidos a procedimientos invasivos o quirúrgicos de tipo programado, existen aspectos muy relevantes con respuestas poco definidas.

Podemos empezar por el servicio médico o facultativo responsable del manejo, antes, durante y después del procedimiento. Cuando se pregunta quién es el responsable del manejo perioperatorio de estos pacientes tratados con AOAD, la respuesta es muy amplia: mayoritariamente son cardiólogos, pero en la extensa lista se incluyen los médicos que realizan la prueba invasiva, médicos de Atención Primaria, hematólogos, farmacéuticos, y enfermeras ${ }^{12}$. En definitiva, en cada centro hospitalario el manejo incumbe a diferentes estamentos, 0 incluso a varios de forma simultánea.

En relación con las guías de práctica clínica y revisiones que abordan el tema que tratamos, existe una variedad nada 
desdeñable de publicaciones. Algunas tratan el tema de una forma general sin especificar patologías ${ }^{13-19}$ y otras detallan actuaciones sólo en pacientes con fibrilación auricular ${ }^{20,21}$. No existe ninguna guía que haga recomendaciones específicas para pacientes con ETV. Respecto a los colectivos médicos responsables de dichas guías, encontramos también diversidad: cirujanos ${ }^{16}$, hematólogos ${ }^{15}$, cardiólogos ${ }^{21}$, anestesistas ${ }^{14,18,22}$; también se han publicado guías donde intervienen en su elaboración varias sociedades científicas en consenso ${ }^{23,24}$. Todas las guías y revisiones a las que nos referimos, sin excepción, tienen el grado de recomendación de consenso de expertos.

Hasta mediados de 2019, las publicaciones disponibles sobre manejo perioperatorio de AOAD, no alcanzan la suficiente calidad como para que las evidencias superen el grado de recomendación de experto. La mayoría de los trabajos son publicaciones con estudios retrospectivos a partir de grandes ensayos clínicos con $A 0 A D$ en pacientes con FANV25,28. Existen escasos trabajos prospectivos, todos con dabigatran y principalmente sobre pacientes sometidos a la colocación de marcapasos ${ }^{29-32}$. Sólo existe una publicación con pacientes diagnosticados de ETV, también retrospectiva y que incluye un número pequeño de casos (190) ${ }^{33}$.

En conjunto, las publicaciones con mayor número de pacientes presentan dos cualidades que les confieren un grado de evidencia bajo a sus resultados: ausencia de un protocolo de manejo unificado para todos y un porcentaje bajo de pacientes sometido a procedimientos de alto riesgo de sangrado (que oscila entre $10 \%$ y el $23 \%$ ) $^{25-28}$, que son aquellos en los que tendríamos que disponer más información por su mayor dificultad de manejo y porque, en definitiva, representan el grupo que más preocupa al médico responsable del procedimiento.

\section{PLANTEAMIENTO GENERAL: RECOMENDACIONES DE LAS GUÍAS}

En todas las guías publicadas hasta la fecha sobre el tema existen coincidencias en el planteamiento general cuyos puntos fundamentales se reducen a responder a tres preguntas: ¿cuándo suspender el tratamiento con AOAD?, ¿se necesita terapia puente?, y ¿cuándo reiniciar el tratamiento después del procedimiento?

El momento de la suspensión del fármaco viene condicionado por dos factores, que son, el aclaramiento de creatinina y el riesgo hemorrágico del procedimiento. Todos los $A O A D$ presentan eliminación renal que varía según el fármaco administrado, así la existencia de niveles de AOAD antes del procedimiento invasivo se relaciona directamente con el funcionamiento renal. En todas las guías, el intervalo de tiempo de suspensión antes del procedimiento es variable según el fármaco y el aclaramiento de creatinina. En 2019 ha sido publicado un documento de consenso de la Sociedad de Trombosis y Hemostasia, donde se establece un listado de procedimientos clasificados según el riesgo hemorrágico ${ }^{34}$.
Se ha dividido en dos grupos: alto riesgo de sangrado (presencia de sangrado mayor en porcentaje superior a $2 \%$ a los 30 días), y bajo riesgo de sangrado (cuando el porcentaje de sangrado mayor es inferior a 2\% a los 30 días). El objetivo principal de este documento de consenso es unificar criterios para futuros trabajos que aborden el tema.

Los resultados del registro de Dresde y del meta-análisis de Nazha et al. ${ }^{35,36}$, ponen de manifiesto que la terapia puente con heparinas de bajo peso molecular (HBPM) en pacientes tratados con $A O A D$, presenta un incremento del riesgo de sangrado mayor, por lo que las guías no recomiendan la utilización de terapia puente. Esta recomendación es unánime, salvo en situaciones muy concretas como son la imposibilidad de tratamiento oral en pacientes con íleo mantenido o situaciones clínicas similares, 0 en aquellos que precisan un nuevo procedimiento quirúrgico o invasivo poco tiempo después del primero21.

A la hora de reiniciar el tratamiento con $A 0 A D$, en la mayoría de las guías se mencionan los parámetros considerados de importancia: el riesgo cardioembólico/tromboembólico y el riesgo hemorrágico del paciente 19,21,22,24. $^{\text {. }}$

Determinadas situaciones como eventos trombóticos recientes, trombofilias de alto riesgo (por ejemplo, síndrome antifosfolípido), $\mathrm{CHA}_{2} \mathrm{DS}_{2}$ VASc superior a 7, 0 cáncer activo, se relacionan con riesgo elevado de eventos ${ }^{34}$. Es por ello que, en la medida de lo posible, se disminuya dicho riesgo, fundamentalmente en base a aumentar el intervalo entre la cirugía/procedimiento programado, y el evento trombótico previo.

En relación con el riesgo hemorrágico en pacientes tratados con AOAD, en los últimos años se están aplicando escalas de riesgo hemorrágico clásicas en pacientes con FANV (HAS-BLED, HEMORR 2 AGHES, y ATRIA) ${ }^{37-39}$. Además, se han desarrollado y validado nuevas escalas a partir de pacientes exclusivamente tratados con AOAD (ORBIT y ABC) ${ }^{40,41}$. Existen publicaciones que comparan las diferentes escalas de riesgo hemorrágico en pacientes con FANV y tratados con AOAD, obteniendo resultados contradictorios o poco clarificadores, y poniendo en evidencia una capacidad predictiva mejorable en prácticamente todas estas escalas ${ }^{40-45}$.

Con todo lo comentado, aún existen aspectos poco claros en el tema. Douketis et al., han demostrado que siguiendo las recomendaciones de suspensión de dabigatran basadas en la cifra de aclaramiento de creatinina, se cuantifican niveles de anticoagulante residual antes del procedimiento, en el $15 \%$ de los pacientes estudiados ${ }^{46}$. Sólo recientemente se ha estandarizado el riesgo hemorrágico de los procedimientos quirúrgicos o pruebas invasivas. Sin embargo no están categorizadas todas ellas, y es prioritario establecer de una forma muy pormenorizada el riesgo hemorrágico de cada uno de los procedimientos. Aunque en las guías y revisiones se hace mención a las escalas de riesgo hemorrágico (HAS-BLED) y riesgo cardioembólico del paciente (CHADS y $\mathrm{CHA}_{2} \mathrm{DS}_{2} \mathrm{VASC}$ ), ninguna de dichas escalas ha sido validada 
en pacientes tratados con $A O A D$ que van a ser sometidos a procedimientos invasivos o quirúrgicos ${ }^{19}$. Este condicionante justifica la ausencia de recomendaciones concretas en las guías, basadas en dichas escalas. Por último, si se revisan las recomendaciones de cada una de las guías publicadas en relación al tema que tratamos, se encuentran discrepancias notorias en temas de extrema importancia, por lo que no se despejan las dudas en el médico responsable para el manejo de estos pacientes. Un ejemplo ilustrativo de esta afirmación es el tiempo que debe ser suspendido dabigatran antes de un procedimiento neuroquirúrgico; dependiendo de las recomendaciones de las sociedades médico-quirúrgicas puede variar entre 5 días, 4-5 días, 204 días, y según la monografía del producto publicado por el fabricante, 2 días ${ }^{18 .}$

\section{ESTUDIO PAUSE}

Con el escenario descrito en párrafos anteriores, la publicación del estudio PAUSE en 2019, ha venido a clarificar muchos aspectos sin respuesta, o con respuestas basadas en opiniones de expertos ${ }^{47}$. El estudio PAUSE es un estudio prospectivo de manejo perioperatorio de AOAD (apixaban, dabigatran y rivaroxaban) en pacientes con FANV, con un protocolo definido de actuación, que incluye suspensión pre-procedimiento, sin terapia puente, y con reinicio postprocedimiento. La hipótesis principal del estudio, establece que con el protocolo definido para cada uno de los AOAD, se consiguen una seguridad (definida por $<2 \%$ de sangrado mayor a los 30 días) y una eficacia (definida por una tasa menor del 1,5\% de eventos cardioembólicos a los 30 días) adecuadas para todos los pacientes. Además, sin necesidad de determinaciones analíticas previas, se asume con este manejo que más del 90\% de los pacientes presentarán niveles mínimos 0 indetectables de AOAD en el momento previo al procedimiento invasivo-quirúrgico. Fueron estudiados 1257 pacientes tratados con apixaban, 668 con dabigatran, y 1082 con rivaroxaban. No existían diferencias significativas entre los grupos al analizar variables clínicas, analíticas, de tratamiento, riesgo hemorrágico del procedimiento, riesgo cardioembólico, ni riesgo hemorrágico de los pacientes. El porcentaje de procedimientos considerados de alto riesgo de sangrado oscilaba entre $32,5 \%$ y $34,5 \%$. Los resultados confirman la seguridad (sangrado mayor a los 30 días: 0,9\%-1,85\%) y la eficacia (eventos tromboembólicos a los 30 días: 0,16\%-0,37\%). En relación con el porcentaje de pacientes con niveles indetectables o mínimos, en los grupos de dabigatran $(92,8 \%-94,4 \%)$ y rivaroxaban $(95,5 \%$ $99,3 \%)$ se cumple la hipótesis del estudio; en el grupo de apixaban ese porcentaje no se cumplía por escaso margen en el grupo de bajo riesgo de sangrado $(87,1 \%)$

Con los resultados de este estudio, muy probablemente se modifiquen o eliminen aspectos asumidos hasta la fecha por las diferentes guías, como son: el aclaramiento de creatinina (que sólo se estudiará en pacientes con dabigatran), el riesgo cardioembólico, y el riesgo hemorrágico del paciente
Sin embargo y contando con que el estudio PAUSE ha dejado claros muchos aspectos, también se pueden extraer una serie de observaciones para la reflexión. Primero, en dicho estudio sólo se han analizado pacientes tratados con dabigatran, apixaban y rivaroxaban, y no existe ningún paciente a tratamiento con edoxaban. En segundo lugar, sólo se incluyen pacientes con fibrilación auricular y no existen pacientes con diagnóstico principal de enfermedad tromboembólica. En tercer lugar, las cifras de los niveles de antiXa y de tiempo de trombina diluido, utilizadas como umbral de seguridad, es decir niveles mínimos o indetectables, no están aceptadas de forma unánime por todos los expertos. Y por último, en la discusión, los autores emplazan a la comunidad investigadora a publicar más estudios, sobre todo en pacientes sometidos a procedimientos de alto riesgo de sangrado, con el objetivo de mejorar el conocimiento en esta área.

\section{CONCLUSIÓN}

Actualmente el manejo perioperatorio programado de pacientes tratados con AOAD es una situación cada vez más frecuente en nuestros hospitales, y con una tendencia al aumento. Existen variedad de guías y revisiones sobre dicho manejo, pero hasta la fecha, las recomendaciones publicadas presentan diferencias significativas según la fuente consultada y con un grado de recomendación basada en expertos. Los resultados del estudio PAUSE han venido a clarificar muchos aspectos, como la falta de necesidad de terapia puente con HBPM, que tampoco es imprescindible el conocimiento del aclaramiento de creatinina del paciente (salvo en aquellos tratados con dabigatran), etc. Esperamos que se sigan publicando más ensayos clínicos sobre el tema, (faltan estudios prospectivos con edoxaban), con el objetivo de mejorar la atención de los pacientes tratados con AOAD que van a ser sometidos a procedimientos invasivos 0 quirúrgicos de una forma programada.

\section{BIBLIOGRAFÍA}

1. Wong S.L., Marshall L.Z., Lawson K.A. Direct oral anticoagulant prescription trends, switching patterns, and adherence in Texas Medicaid. Am J Manag Care. 2018;24:SP309SP314.

2. Ibáñez L, Sabaté M, Vidal X, Ballarin E, Rottenkolber M, Schmiedl S, Heeke A, Huerta C, Martin Merino E, Montero D, Leon-Muñoz LM, Gasse C, Moore N, Droz C, Lassalle R, Aakjaer M, Andersen M, De Bruin ML, Groenwold R, van den Ham HA, Souverein P, Klungel 0 , Gardarsdottir $H$. Incidence of direct oral anticoagulant use in patients with nonvalvular atrial fibrillation and characteristics of users in 6 European countries (2008-2015): A crossnational drug utilization study. Br J Clin Pharmacol. 2019; 85:2524-2539.

3. Rodwin BA, Salami JA, Spatz ES, Valero-Elizondo J, Virani SS, Blankstein R, Blaha MJ, Nasir K, Desai NR. Variation in the Use of Warfarin and Direct Oral Anticoagulants in Atrial Fibrillation and Associated Cost Implications. Am J Med. 2019;132:61-70.

4. Steinberg BA, Gao H, Shrader P, Pieper K, Thomas L, Camm AJ, Ezekowitz MD, Fonarow GC, Gersh BJ, Goldhaber S, Haas S, Hacke W, Kowey PR, Ansell J, Mahaffey KW, Naccarelli G, Reiffel JA, Turpie A, Verheugt F, Piccini JP, Kakkar A, Peterson ED, Fox KAA; GARFIELDAF: ORBIT-AF Investigators. International trends in clinical characteristics and oral anticoagulation treatment for patients with atrial fibrillation: Results from the GARFIELD-AF, ORBIT-AF I, and ORBIT-AF II registries. Am Heart J. 2017;194:132-140.

5. Kirchhof $P$, Benussi S, Kotecha D, Ahlsson A, Atar D, Casadei B, Castella M, Diener HC, Heidbuchel H, Hendriks J, Hindricks G, Manolis AS, Oldgren J, Popescu BA, Schotten U, Van Putte B, Vardas P; ESC Scientific Document Group. 2016 ESC Guidelines for the management of atrial fibrillation developed in collaboration with EACTS. Eur Heart J. 2016;37:2893-2962.

6. Kearon C, AkI EA, Ornelas J, Blaivas A, Jimenez D, Bounameaux H, Huisman M, King CS, Morris TA, Sood N, Stevens SM, Vintch JRE, Wells P, Woller SC, Moores L. Antithrombotic 
Therapy for VTE Disease: CHEST Guideline and Expert Panel Report. Chest. 2016; 149:315352.

7. Silverstein MD, Heit JA, Mohr DN, Petterson TM, O'Fallon WM, Melton LJ 3rd. Trends in the incidence of deep vein thrombosis and pulmonary embolism: a 25-year population-based study. Arch Intern Med. 1998;158:585-93.

8. Guijarro Merino R, Montes Santiago J, San Román Terán CM. Epidemiología de la enfermedad tromboembólica venosa en España. Med Clin (Barc). 2008;131 (Suppl 2):S2 S9.

9. Hanon O, Assayag P, Belmin J, Collet JP, Emeriau JP, Fauchier L, Forette F, Friocourt P, Gentric A, Leclercq C, Komaida M, Le Heuzey JY; French Society of Geriatrics and Gerantology; French Society of Cardiology. Expert consensus of the French Society of Geriatrics and Gerontology and the French Society of Cardiology on the management of atrial fibrillation in elderly people. Arch Cardiovasc Dis. 2013;106:303-23.

10. population.un.org [internet] United Nations Department of Economic and Social Affairs. Dispoible en: https://population.un.org/wpp/Maps/. Acceso 26/11/2019.

11. Douketis JD, Berger PB, Dunn AS, Jaffer AK, Spyropoulos AC, Becker RC, Ansell J. The perioperative management of antithrombotic therapy: American College of Chest Physicians Evidence-Based Clinical Practice Guidelines (8th Edition). Chest. 2008;133(Suppl 6):S299-S339.

12. Flaker GC, Theriot P, Binder LG, Dobesh PP, Cuker A, Doherty JU. Management of Periprocedural Anticoagulation: A Survey of Contemporary Practice. J Am Coll Cardiol. 2016;68:217-26.

13. Steffel J., Verhamme P., Potpara T.S., Albaladejo P., Antz M., Desteghe L., Haeusler K.G., Oldgren J., Reinecke H., Roldan-Schilling V., Rowell N., Sinnaeve P., Collins R., Camm A.J., Heidbüchel H.; ESC Scientific Document Group. The 2018 European Heart Rhythm Association Practical Guide on the use of non-vitamin K antagonist oral anticoagulants in patients with atrial fibrillation. Eur Heart J. 2018;39:1330-1393.

14. Porter J, Dinsmore J. Perioperative Management of Direct Oral Anticoagulants in Intracranial Surgery. J Neurosurg Anesthesiol. 2020;32(4):300-306.

15. Keeling D, Tait RC, Watson H; British Committee of Standards for Haematology. Perioperative management of anticoagulation and antiplatelet therapy. Br J Haematol. 2016; 175:602-613.

16. Hornor MA, Duane TM, Ehlers AP, Jensen EH, Brown PS Jr, Pohl D, da Costa PM, Ko CY, Laronga C. American College of Surgeons' Guidelines for the Perioperative Management of Antithrombotic Medication. J Am Coll Surg. 2018;227:521-536.

17. Sunkara T, Ofori E, Zarubin V, Caughey ME, Gaduputi V, Reddy M. Perioperative Management of Direct Oral Anticoagulants (DOACs): A Systemic Review. Health Serv Insights. 2016; (Suppl 1):S25-S36.

18. Leitch J, van Vlymen J. Managing the perioperative patient on direct oral anticoagulants. Can J Anaesth. 2017;64:656-672.

19. Dubois V, Dincq AS, Douxfils J, Ickx B, Samama CM, Dogné JM, Gourdin M, Chatelain B, Mullier F, Lessire S. Perioperative management of patients on direct oral anticoagulants. Thromb J. 2017; 15:14

20. Omae T, Koh K, Kumemura M, Sakuraba S, Katsuda Y. Perioperative management of patients with atrial fibrillation receiving anticoagulant therapy. J Anesth. 2019; 33:551-561.

21. Doherty JU, Gluckman TJ, Hucker WJ, Januzzi JL Jr, Ortel TL, Saxonhouse SJ, Spinler SA 2017 ACC Expert Consensus Decision Pathway for Periprocedural Management of Anticoagulation in Patients With Nonvalvular Atrial Fibrillation: A Report of the American College of Cardiology Clinical Expert Consensus Document Task Force. J Am Coll Cardiol. 2017; 69:871-898.

22. Yurttas T, Wanner PM, Filipovic M. Perioperative management of antithrombotic therapies. Curr Opin Anaesthesiol. 2017;30:466-473.

23. Prisco D, Ageno W, Becattini C, D’Angelo A, Davi G, De Cristofaro R, Dentali F, Di Minno G, Falanga A, Gussoni G, Masotti L, Palareti G, Pignatelli P, Santi RM, Santilli F, Silingard M, Tufano A, Violi F; SIMI (Italian Society of Internal Medicine); FADOI (Federation of Associations of Hospital Doctors on Internal Medicine); SISET (Italian Society for the Study of Haemostasis and Thrombosis). Italian intersociety consensus on DOAC use in internal medicine. Intern Emerg Med. 2017; 12:387-406.

24. Vivas D, Roldán I, Ferrandis R, Marín F, Roldán V, Tello-Montoliu A, Ruiz-Nodar JM, GómezDoblas JJ, Martín A, Llau JV, Ramos-Gallo MJ, Muñoz R, Arcelus JI, Leyva F, Alberca F, Oliva R, Gómez AM, Montero C, Arikan F, Ley L, Santos-Bueso E, Figuero E, Bujaldón A, Urbano J, Otero R, Hermida JF, Egocheaga I, Llisterri JL, Lobos JM, Serrano A, Madridano O, Ferreiro $\mathrm{JL}$; Expert reviewers. Perioperative and Periprocedural Management of Antithrombotic Therapy: Consensus Document of SEC, SEDAR, SEACV, SECTCV, AEC, SECPRE, SEPD, SEGO, SEHH, SETH, SEMERGEN, SEMFYC, SEMG, SEMICYUC, SEMI, SEMES, SEPAR, SENEC, SEO, SEPA, SERVEI, SECOT and AEU. Rev Esp Cardiol. 2018; 71:553-564.

25. Healey JS, Eikelboom J, Douketis J, Wallentin L, Oldgren J, Yang S, Themeles E, Heidbuchel $H$, Avezum A, Reilly P, Connolly SJ, Yusuf S, Ezekowitz M; RE-LY Investigators. Periprocedural bleeding and thromboembolic events with dabigatran compared with warfarin: results from the Randomized Evaluation of Long-Term Anticoagulation Therapy (RE-LY) randomized trial. Circulation. 2012; 126:343-8.

26. Sherwood MW, Douketis JD, Patel MR, Piccini JP, Hellkamp AS, Lokhnygina Y, Spyropoulos AC, Hankey GJ, Singer DE, Nessel CC, Mahaffey KW, Fox KA, Califf RM, Becker RC; ROCKET AF Investigators. Outcomes of temporary interruption of rivaroxaban compared with warfarin in patients with nonvalvular atrial fibrillation: results from the rivaroxaban once daily, oral, direct factor Xa inhibition compared with vitamin $\mathrm{K}$ antagonism for prevention of stroke and embolism trial in atrial fibrillation (ROCKET AF). Circulation. 2014; 129:1850-9.

27. Douketis JD, Murphy SA, Antman EM, Grip LT, Mercuri MF, Ruff CT, Weitz Jl, Braunwald E, Giugliano RP. Peri-operative Adverse Outcomes in Patients with Atrial Fibrillation Taking Warfarin or Edoxaban: Analysis of the ENGAGE AF-TIMI 48 Trial. Thromb Haemost. 2018; 118:1001-1008.
28. Garcia D, Alexander JH, Wallentin L, Wojdyla DM, Thomas L, Hanna M, Al-Khatib SM, Dorian P, Ansell J, Commerford P, Flaker G, Lanas F, Vinereanu D, Xavier D, Hylek EM, Held C, Verheugt FW, Granger CB, Lopes RD. Management and clinical outcomes in patients treated with apixaban vs warfarin undergoing procedures. Blood. 2014; 124:3692-8.

29. Schulman S, Carrier M, Lee AY, Shivakumar S, Blostein M, Spencer FA, Solymoss S, Barty R, Wang G, Heddle N, Douketis JD; Periop Dabigatran Study Group. Perioperative Management of Dabigatran: A Prospective Cohort Study. Circulation. 2015; 132:167-73.

30. Kosiuk J, Koutalas E, Doering M, Nedios S, Sommer P, Rolf S, Darma A, Breithardt OA, Dinov $B$, Hindricks G, Richter S, Bollmann A. Comparison of dabigatran and uninterrupted warfarin in patients with atrial fibrillation undergoing cardiac rhythm device implantations. Casecontrol study. Circ J. 2014;78:2402-2407.

31. Madan S, Muthusamy P, Mowers KL, Elmouchi DA, Finta B, Gauri AJ, Woelfel AK, Fritz TD, Davis AT, Chalfoun NT. Safety of anticoagulation with uninterrupted warfarin vs. interrupted dabigatran in patients requiring an implantable cardiac device. Cardiovasc Diagn Ther. 2016; 6:3-9.

32. Terekhov D, Agapov V, Kulikov K, Zadorozhnaya S, Samitin V, Maslyakov V. Pacemaker Implantation in Elderly Patients: Safety of Various Regimens of Anticoagulant Therapy. J Atr Fibrillation. 2017; 9:1467.

33. Shaw J, de Wit C, Le Gal G, Carrier M. Thrombotic and bleeding outcomes following perioperative interruption of direct oral anticoagulants in patients with venous thromboembolic disease. J Thromb Haemost. 2017; 15:925-930.

34. Spyropoulos AC, Brohi K, Caprini J, Samama CM, Siegal D, Tafur A, Verhamm P, Douketis JD; SSC Subcommittee on Perioperative and Critical Care Thrombosis and Haemostasis of the International Society on Thrombosis and Haemostasis. Scientific and Standardization Committee Communication: Guidance document on the periprocedural management of patients on chronic oral anticoagulant therapy: Recommendations for standardized reporting of procedural/surgical bleed risk and patient-specific thromboembolic risk. J Thromb Haemost. 2019; 17:1966-1972.

35. Beyer-Westendorf J, Gelbricht V, Förster K, Ebertz F, Köhler C, Werth S, Kuhlisch E, Stange T, Thieme C, Daschkow K, Weiss N. Peri-interventional management of novel oral anticoagulants in daily care: results from the prospective Dresden NOAC registry. Eur Heart J. 2014;35:1888-96

36. Nazha B, Pandya B, Cohen J, Zhang M, Lopes RD, Garcia DA, Sherwood MW, Spyropoulos AC. Periprocedural Outcomes of Direct Oral Anticoagulants Versus Warfarin in Nonvalvular Atrial Fibrillation. Circulation. 2018; 138:1402-1411.

37. Pisters R., Lane D.A., Nieuwlaat R., de Vos C.B., Crijns H.J., Lip G.Y. A novel user-friendly score (HAS-BLED) to assess 1-year risk of major bleeding in patients with atrial fibrillation: the Euro Heart Survey. Chest. 2010; 138:1093-1100.

38. Gage B.F., Yan Y., Milligan P.E., Waterman A.D., Culverhouse R., Rich M.W., Radford M.J. Clinical classification schemes for predicting hemorrhage: results from the National Registry of Atrial Fibrillation (NRAF). Am Heart J. 2006;151:713-719.

39. Fang M.C., Go A.S., Chang Y., Borowsky L.H., Pomernacki N.K., Udaltsova N., Singer D.E. A new risk scheme to predict warfarin-associated hemorrhage: The ATRIA (Anticoagulation and Risk Factors in Atrial Fibrillation) Study. J Am Coll Cardiol. 2011; 58:395-401.

40. O'Brien EC, Simon DN, Thomas LE, Hylek EM, Gersh BJ, Ansell JE, Kowey PR, Mahaffey KW, Chang P, Fonarow GC, Pencina MJ, Piccini JP, Peterson ED. The ORBIT bleeding score: a simple bedside score to assess bleeding risk in atrial fibrillation. Eur Heart $\mathrm{J}$. 2015;36:3258-3264.

41. Hijazi Z., Oldgren J., Lindbäck J., Alexander JH, Connolly SJ, Eikelboom JW, Ezekowitz MD, Held C, Hylek EM, Lopes RD, Siegbahn A, Yusuf S, Granger CB, Wallentin L; ARISTOTLE and RE-LY Investigators. The novel biomarker-based ABC (age, biomarkers, clinical history)bleeding risk score for patients with atrial fibrillation: a derivation and validation study. Lancet. 2016; 387:2302-2311.

42. Proietti M, Hijazi Z., Andersson U, Connolly SJ, Eikelboom JW, Ezekowitz MD, Lane DA, Oldgren J, Roldan V, Yusuf S, Wallentin L; RE-LY Investigators. Comparison of bleeding risk scores in patients with atrial fibrillation: insights from the RE-LY trial. J Intern Med. 2018;283:282-292.

43. Yao X, Gersh BJ, Sangaralingham LR, Kent DM, Shah ND, Abraham NS, Noseworthy PA. Comparison of the $\mathrm{CHA}_{2} \mathrm{DS}_{2}$-VASc, CHADS, HAS-BLED, ORBIT, and ATRIA Risk Scores in Predicting Non-Vitamin K Antagonist Oral Anticoagulants-Associated Bleeding in Patients With Atrial Fibrillation. Am J Cardiol. 2017; 120:1549-1556.

44. Lip GYH, Skjøth F, Nielsen PB, Kjældgaard JN, Larsen TB. The HAS-BLED, ATRIA, and ORBIT Bleeding Scores in Atrial Fibrillation Patients Using Non-Vitamin K Antagonist Oral Anticoagulants. Am J Med. 2018;131:574.e13-574.e27.

45. Caro Martínez C, Andreu Cayuelas JM, Flores Blanco PJ, Valdés M, Bailén Lorenzo JL, Manzano Fernández S. Comparison of Bleeding Risk Scores in Patients With Nonvalvular Atrial Fibrillation Starting Direct Oral Anticoagulants. Rev Esp Cardiol. 2017; 70:878-880.

46. Douketis JD, Wang G, Chan N, Eikelboom JW, Syed S, Barty R, Moffat KA, Spencer FA, Blostein M, Schulman S. Effect of standardized perioperative dabigatran interruption on the residual anticoagulation effect at the time of surgery or procedure. J Thromb Haemost. 2016; 14:89-97.

47. Douketis JD, Spyropoulos AC, Duncan J, Carrier M, Le Gal G, Tafur AJ, Vanassche T, Verhamme P, Shivakumar S, Gross PL, Lee AYY, Yeo E, Solymoss S, Kassis J, Le Templier G, Kowalski S, Blostein M, Shah V, MacKay E, Wu C, Clark NP, Bates SM, Spencer FA, Arnaoutoglou E, Coppens M, Arnold DM, Caprini JA, Li N, Moffat KA, Syed S, Schulman S. Perioperative Management of Patients With Atrial Fibrillation Receiving a Direct Oral Anticoagulant. JAMA Intern Med. 2019;179(11):1469-1478. 